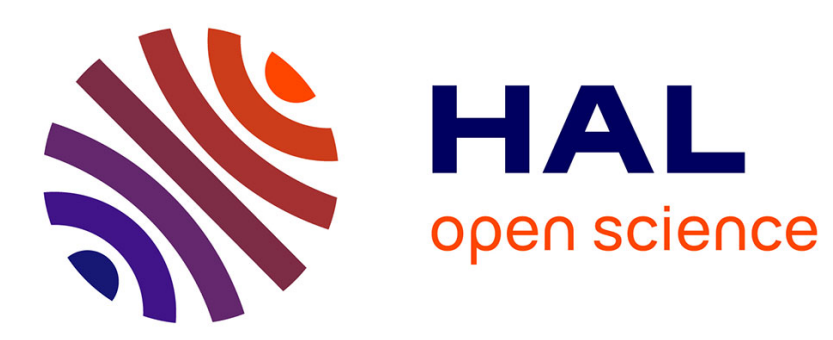

\title{
La réapparition du mot humus au XVIIIe siècle et sa signification agronomique
}

\author{
C. Feller, Jean Boulaine
}

\section{To cite this version:}

C. Feller, Jean Boulaine. La réapparition du mot humus au XVIIIe siècle et sa signification agronomique. Revue forestière française, 1987, 39 (6), pp.487-495. 10.4267/2042/25821. hal03424720

\section{HAL Id: hal-03424720 \\ https://hal.science/hal-03424720}

Submitted on 10 Nov 2021

HAL is a multi-disciplinary open access archive for the deposit and dissemination of scientific research documents, whether they are published or not. The documents may come from teaching and research institutions in France or abroad, or from public or private research centers.
L'archive ouverte pluridisciplinaire HAL, est destinée au dépôt et à la diffusion de documents scientifiques de niveau recherche, publiés ou non, émanant des établissements d'enseignement et de recherche français ou étrangers, des laboratoires publics ou privés. 


\title{
biologie et forêt
}

\section{LA RÉAPPARITION DU MOT HUMUS AU XVIII ${ }^{e}$ SIĖCLE ET SA SIGNIFICATION AGRONOMIQUE}

\author{
C. FELLER - J. BOULAINE
}

Dans leur "Dictionnaire des sols", Plaisance et Cailleux (1958), à l'article " Humus ", écrivent: ..." par une étrange évolution le mot humus a vu son sens se restreindre de "terre " en général au sens "matière organique incorporee au sol" ". C'est l'histoire de cette "ètrange évolution" qui, à l'aide de documents bibliographiques des XVIII et $X I X^{e}$ siècles, est retracèe ici.

Cette recherche historique, sans être exhaustive, a permis de nombreux recoupements, en particulier avec les doctrines agricoles ${ }^{(1)}$ affichèes au cours de cette période.

Pour les vieux auteurs latins, le mot humus signifie sol. Ainsi Columelle : "il faut pour le froment deux pieds de bon humus " (note 9 page 61 de l'édition de 1804 du livre de O. de Serre; la note a étè rédigée par François de Neufchateau).

Avant notre ère, le mot humus signifie donc à peu près: "terre végétale ".

Les principes nutritifs des végétaux, quand il en est question, sont alors imaginès comme une huile ou une graisse "oleum onctuosum" ou "terrae adeps" d'où l'utilisation du terme engrais que nous employons toujours et des expressions comme "terre de vieille graisse" ou autres.

La liaison avec la teneur en humus n'est pas générale et certains auteurs pensent que la graisse du sol provient de la marne (d'après Giesecke, 1929, p. 36).

Selon Martin (1941), le mot latin humus avec sa signification de "sol " a été progressivement abandonné après Cicéron (106-43 av. J.C.) et remplacé par "terra", ce qui a conduit, en français, jusqu'au XVIII" siècle, à l'utilisation des mots "terre ", "terreau ", " terre végètale ". Par

(1) Le lecteur intéressé pourra se référer aux ouvrages de Bourde (1962) et Godard (s.d.) pour une connaissance détaillée de ces doctrines agricoles au XVIII ${ }^{e}$ siécle. 


\section{FELLLER - J. BOULAINE}

contre, "inhumer ", mettre en terre, est attesté en 1413 et ses dérivés inhumation, exhumation, exhumer ont été utilisés à l'époque classique.

Humus (Robert, 1966 ; Trésor de la Langue française, 1981) apparaît en 1765 au tome 8 de l'Encyclopédie: "Humus, Histoire naturelle, les naturalistes empruntent souvent ${ }^{(2)}$ ce mot latin, même en français, pour désigner le terreau, la terre des jardins, ou la terre formée par la décomposition des végétaux; c'est la terre ${ }^{(3)}$ brune ou noirâtre qui est à la surface de la terre. Voire terre végétale et terreau".

Nous allons voir toutefois que:

- le terme humus ne passe pas immédiatement dans le langage scientifique (naturaliste ou agricole), ce qui implique qu'il était relativement peu usité à cette époque;

- sa définition d'horizon humifère, telle qu'elle ressort de l'Encyclopédie, est loin d'être bien assimilée, humus pouvant signifier indifféremment, sol, horizon humifère ou constituant organique du sol sans aucune précision des auteurs.

Notre analyse bibliographique s'organise autour de trois dates repères correspondant à des parutions d'ouvrages largement diffusés:

- 1765 : l'Encyclopédie de Diderot et d'Alembert pour l'ancienneté de la citation du mot humus :

- 1781: premier tome du "Cours complet d'agriculture" de l'abbé Rozier, véritable encyclopédie de l'agriculture ;

- 1809: première édition en allemand des "Principes raisonnés d'agriculture " (traduit en français ä partir de 1811) de Thaer, agronome allemand, père de la "Théorie de I'humus", théorie qui marquera profondément les doctrines agricoles de la première moitié du XIX siécle.

\section{AVANT 1783 - LE MOT HUMUS N'EST PRATIQUEMENT PAS UTILISÉ}

Avant 1765, le mot humus n'est cité dans pratiquement aucun ouvrage. II n'est employé ni par l'Abbé de Vallemont dans "Curiosités de la Nature" (anonyme 1735), ni dans la "Nouvelle Maison Rustique" (Liger, 1721 et 1749), ni dans " l'Histoire naturelle générale et particulière..." (Buffon, 1752), ni dans les "Principes de l'agriculture et de la végétation" (Home, 1761), ni dans les "Éléments d'agriculture» de Duhamel du Monceau (1763), ni dans "Contemplation de la nature " (Bonnet, 1764), ni dans " Histoire de l'agriculture ancienne" (anonyme, 1765). Tous ces auteurs attribuent la fertilité du sol à des "sels", des "huiles", un "limon subtil onctueux et salin ", à des "substances bitumineuses".

Les seuls à citer le mot humus en tant que terme latin, sont Wallerius ("Minéralogie", 1753, tome 1, pp. XXIV et 9-19) comme équivalent de "terre de jardin ", "terre pourrie ", Linné (et encore n'est-ce pas sûr) qui parle du cycle de l'humus entre le sol, la plante, les animaux et I'homme, et Valmont de Bomare dans son "Dictionnaire d'histoire naturelle" (1768) avec le sens de sol ou d'horizon de sol.

Humus n'est pas davantage utilisé entre 1765 et 1783 . II n'en est pas fait mention dans le "Manuel d'Agriculture pour le laboureur" (La Salle de l'Etang, 1768), ni dans "...les caractères principaux des terres en général " (Bergmann, 1773), ni dans le "Bon jardinier" (De Grace,

(2) La suite nous montrera que ce n'est pas si " souvent " que cela!

(3) II faut signaler aussi l'ambiguitè qui pèse sur le mot " terre", utilisè, soit, dans le sens de matériau terrigène ou de sol, soit, très souvent (langage des chimistes) comme constituant minéral. Exemple: "terre calcaire" peut aussi bien signifier "sol calcaire "que " carbonate de calcium ". 
1783), ni dans Delaillevault (1783) avec ses "Recherches sur les houilles d'engrais" (4), ni dans le "Dictionnaire de l'Académie française" (1777). Si Fabroni, citè par Bourde, est le premier à insister sur l'importance de la "terre végétale" comme principe de la fertilité des sols, ni l'un ni l'autre n'emploient le mot humus.

En conclusion, si humus est, dans quelques rares cas, parfois cité au cours de la période 17111781, c'est presque toujours en tant que mot latin avec la signification de "terre" ou " terre végétale ", la notion de constituant n'étant pas clairement exprimée.

\section{DE 1781 À 1809 - HUMUS PASSE DU LATIN AU FRANÇAIS}

L'édition du "Cours complet d'agriculture "... de l'Abbé Rozier s'étalera, suppléments compris, sur 24 ans (1781-1805) et ne sera d'ailleurs achevée qu'après sa mort (dans son lit, par une bombe, lors du siège de Lyon), en 1793, par une "société de savants et d'agriculteurs" ${ }^{(5)}$. Cet ouvrage, qui se présente comme un dictionnaire, ne traite pas du mot humus à la lettre $\mathrm{H}$. II faut croire que l'humus n'a pas encore une place suffisamment importante et reconnue dans les doctrines agricoles de l'époque pour être répertorié en tant que tel. Ce mot apparaît pourtant à divers endroits de l'ouvrage et ceci dès le tome 1. Quelques exemples:

- 1781, tome 1, pp. 478-502 à « Amender, Amendement... dans tous les amendements... on doit se proposer... $1^{\circ} \ldots 2^{\circ}$ à créer le terreau ou humus dans la plus grande quantité possible parce que le terreau est la seule terre végétale... ";

- 1781, tome 1, p. 627 à "Arbre... ce sont les arbres qui ont insensiblement prèparè la terre que nous cultivons. Elle doit à leurs débris entassés pendant une longue suite de siècles, cet humus ou terre végétale qui assure l'abondance des moissons... Abattez une forêt... semez continuellement sur ce terrain, peu à peu les récoltes absorberont la terre végétale, les pluies en entraineront le reste... Ce sol auparavant noir et fertile changera de couleur, il ne restera plus qu'un grain de terre sec, aride et granuleux...";

- 1796, tome 9, pp. 390-401 à "Terre... la terre calcaire est donc la seule terre végétale, le véritable humus soluble dans l'eau et la seule qui établisse et constitue la charpente des plantes... si on amoncelle les plantes... si on les laisse se décomposer... on obtiendra en dernière analyse, la terre calcaire pure, le vèritable humus... Cultivateurs ne songez qu'à créer ce précieux humus... qui est une vraie terre animalisée... la seule qui entre dans leur composition... " (les plantes).

Tous les articles cités sont de l'Abbé Rozier (sauf terre qui est peut-être d'un de ses continuateurs ?). On constate par rapport aux ouvrages des périodes précédentes une évolution très nette des concepts de fertilité et de nutrition des plantes qui annonce déjà la "théorie de l'humus ". Si la définition de l'humus oscille toujours entre celles d'horizon et de constituant, l'humus est déjà le principe de végétation, c'est "la seule terre " qui entre dans la composition des plantes.

Une étape supplémentaire ${ }^{(6)}$ est franchie par Hassenfratz qui publie, en 1792, trois mémoires "Sur la nutrition des végétaux". Dans les deux premiers, il s'évertue, à l'aide de diverses expériences et affirmations, à démontrer la fausseté des théories récentes du rôle de l'air dans la nutrition carbonée des végétaux, en particulier celle d'Ingen-Housz (1780). II écrit alors dans

(4) Ouvrage par ailleurs tout à fait intèressant quant à la classification des sols proposèe par l'auteur, très èlaborèe pour l'époque.

(5) Parmi lesquels on peut citer : Parmentier, Chaptal, De Candolle, Yvart, Thouin, Bosc...

(6) En ce qui concerne l'analyse immediate ou totale de l'humus, Achard (1786), Vauquelin (1797), de Saussure (1804), Thomson (1807) contribuérent à mettre au point l'extraction de l'humus et de certaines de ses fractions (ulmine, humic extract...). 
le troisième mémoire: ... "Concluons que de toutes les manières d'expliquer l'accroissement du carbone dans les plantes par l'acte de la végétation, celle qui a un rapport plus direct avec les engrais, celle qui s'accorde le mieux avec tous les faits connus, est la dissolution du charbon dans l'eau, sucé ensuite par les racines et déposé dans l'intérieur des plantes; qu'ainsi, le charbon dissous dans l'eau est une des substances nutritives des plantes".

Ainsi, avec Hassenfratz, la "terre" qui entre dans la composition des plantes est le "charbon". Les fondements théoriques de la "Théorie de l'humus" sont posés.

Toutefois, au cours de cette pèriode $(1780-1810)$, le débat reste très ouvert comme en témoignent les exemples ci-dessous:

- Dans le “Nouveau dictionnaire d'histoire nature/le", à l'article de Patrin (1802, tome 11, pp. 445-447) : "Humus, Terre végétale ou Terreau ", l'auteur affirme très clairement que ce n'est pas l'humus qui nourrit la plante mais l'atmosphere: "... on sait que ce n'est pas aux dejpens du sol mais de l'atmosphère que les végètaux prennent leur accroissement. Mille expériences en ont fourni la preuve directe" (7). Par contre, l'auteur donne le sens de "Sol " à l'humus: " c'est la couche la plus extérieure de la terre, celle qui forme le sol de toutes les contrées du monde partout où le roc n'est pas à découvert: son épaisseur varie depuis deux ou trois doigts jusqu'à plusieurs pieds...".

- Dans le même ouvrage, Virey, à l'article “Alimens" (1803, tome 1, pp. 222-233), affiche une opinion totalement opposée quant à la nutrition végétale : ce ne sont ni la terre, ni l'eau, ni l'air qui nourrissent la plante mais l'humus "débris de corps organisés " selon le principe que seules les substances qui ont été vivantes et organisèes peuvent être des substances nourricieres. Remarquons qu'ici Humus est utilisé dans le sens de constituant.

- De Saussure (1804) dans ses "Recherches chimiques sur la végétation" prend une position intermédiaire entre ce que seront la "Théorie de l'humus" et la "Thèorie minérale " mais en ètant beaucoup plus proche de cette derniere. Dès les premières pages de son remarquable livre ("Avertissement" $\mathrm{p}$. VI) ..." Mes recherches me conduisent à montrer comment l'eau et l'air contribuent plus à la formation de la substance sèche des plantes... que la matière même du terreau qu'elles absorbent en dissolution par leurs racines... ". Aux pages 266 à 271, il reprend ce problème en signalant que la proportion de matière sèche de la plante provenant du terreau ne représente qu'environ $1 / 20^{e}$ (p. 269) de celle fournie par l'atmosphère. Au regret de tous les historiens des doctrines agricoles (cf. par exemple Grandeau, $1879^{(8)}$ ), De Saussure n'a pas "franchi le pas" qui l'aurait conduit avec quarante ans d'avance sur Liebig et Boussingault à développer la "Théorie minérale" de la nutrition végétale.

Il est d'autres ouvrages généraux sur l'agriculture de la même période qui ne citent toujours pas le mot Humus (Yvart, 1807 ; Douette-Richardot, 1806).

Concernant la signification attribuée au mot Humus nous retiendrons donc de cette expérience que si Humus est principalement pris dans le sens d'horizon organique ou organo-minéral, il est parfois utilisé dans le sens de sol (exemple Patrin), ou même associé à des notions curieuses telles que "limon " = "humus " ou " terre calcaire " = "véritable humus ", ce qui confirme bien le "flou " existant encore autour de cette notion. Rien d'ètonnant quand on constate qu'en 1823 un scientifique aussi reconnu que Chaptal parle d' Humus minéral" dans sa "Chimie appliquée à l'agriculture " (tome 1, p. 43).

(7) En particulier les travaux de Priestley (1777), Senebier (1782), Ingen-Housz (1780).

(8) p. 46 : "Tout en edifiant, par ses travaux, la doctrine de /'humus, Th. de Saussure a decouvert de nombreux faits sur lesquels repose en partie la thérie de la nutrition des plantes: mais pas plus que Davy, if n'en a tiré de conclusions relativement à l'épuisement du sol..." 


\section{FELLER - J. BOULAINE}

sa nature, et en général il est assez semblable à lui-même. C'est un produit de la force organique, une combinaison de carbone, d'hydrogène, d'azote et d'oxygène... A ces substances essentielles de l'humus, il s'en joint encore quelques autres en plus petite quantité, du phosphore, du soutre, un peu de terre proprement dite, et quelquefois différents sels...".

p. 104 : "L'humus a de l'analogie avec les corps dont il est le produit, quant à la qualité de ses parties constituantes, mais ces parties y éprouvent un changement dans leurs quantités respectives. Les substances élémentaires entrent dans une nouvelle combinaison, il s'en évapore une partie. Suivant de Saussure, l'humus contient moins d'oxygène, mais plus de carbone et d'azote que les végétaux dont il a été tiré. Mais les circonstances sous lesquelles l'humus se forme ont sans doute une grande influence sur les proportions de ses éléments et sur les divers genres de combinaisons de ses parties élémentaires... Cela est démontré quoique, ni les circonstances qui ont de l'intluence sur la formation de l'humus, ni les déviations auxquelles elles sont soumises, n'aient pas encore été sutfisamment analysées... .

p. 105 : “ D’après les expériences faites sous le récipient, on peut calculer quelle énorme quantité de gaz acide carbonique doit se dégager d'un journal de terre riche en humus... Dans le même temps, l'humus éprouve encore un autre changement, que de Saussure nous a également appris à connaître d'une maniẻre plus particulière. II s'y forme une certaine matière qui est soluble dans l'eau, et qu'on nomme matière extractive... Suivant de Saussure, l'humus qui est ainsi privé de sa matière extractive soluble, est moins técond, il contient proportionnément moins de carbone que celui qui n'a pas été soumis à la coction. De Saussure vit la matière extractive détrempée dans l'eau, passer immédiatement dans les racines des plantes; il paraît donc que cette substance est, après l'acide carbonique, une des matières les plus propres à introduire des aliments, et en particulier du carbone, dans les suçoirs des plantes. On n'obtient que peu de matière extractive du vieux humus par la simple pression, et à moins de lui faire subir une coction; en revanche, on en obtient davantage de I'humus récent ou mêlé d'engrais animaux... Les alkalis fixes dissolvent presqu'entièrement tant d'humus que cette partie de la matière extractive qui était ainsi devenue insoluble; pendant leur action. il se dégage de l'ammoniaque. Cette dissolution est décomposée par les acides, qui en précipitent une poudre intiammable, mais dont la quantité est petite, en proportion de celle de l'humus. L'alcool ne dissout pas l'humus; il n'en sépare qu'un peu de matière extractive et de résine... ».

On pourrait continuer longuement sur ces citations de Thaer avec des observations sur les propriétés de l'humus dans les terrains sableux, hydromorphes, tourbeux, sur son caractère acide, acidité fonction du couvert végétal, sur la comparaison des humus d'origine végétale ou animale, ce dernier étant plus riche en azote et phosphore, sur son rôle dans l'amélioration des terrains argileux, etc... et de conclure : "... nous avons besoin de recherches pneumatiques sur l'humus, plus précises que celles qui nous sont connues, pour pouvoir tixer les proportions des diverses parties intégrantes qui entrent dans ses diverses espèces...".

II nous faut remarquer que, probablement pour la première fois (?), le terme humus est restreint, et de manière très précise ${ }^{(11)}$ à la notion de constituant, constituant dont les propriétés sont largement développées par l'auteur. A l'exception de ce qui ressort de la nutrition végétale, cette présentation apparaît très complète pour l'époque et encore acceptable de nos jours.

Ainsi, si Thaer est en recul sur certains de ses contemporains ${ }^{(12)}$ pour ses théories sur la nutrition végétale, son ouvrage marque par contre un tournant décisif dans la perception de l'humus comme constituant organique des sols.

C'est à la même époque que naît la chimie de l'humus (cf. Kononova, 1961, chapitre 1). Pourtant les termes chimiques dérivés du mot humus (humine, acide humique) ne semblent apparaître

(11) Ce souci de précision du vocabulaire utilise se retrouve tout au long de l'ouvrage, par exemple, pour les mots "terre ", "agriculture ", "agronomie ", etc.

(12) De Saussure, en particulier, qu'il interprète exagèrément, selon ses propres hypothéses. 
que plus tardivement (vers 1820) et curieusement postérieurement à ceux d'ulmine ${ }^{(13)}$ et acides ulmiques qui ont donc servi à désigner pendant une période assez longue les substances humiques du sol. En effet, selon Maillard (1913, p. 365), " acide humique" serait une expression de Dobereiner dont les travaux, cités par ailleurs dans le texte (p. 356) sont de 1823.

II n'est donc pas impossible que la notion d'humus = constituant ne fut pas suffisamment implantée dans les esprits avant 1809 pour que puissent apparaître les qualificatifs "humique" ou "humine" désignant les substances humiques du sol.

\section{APRÈS THAER}

La "Théorie de l'humus" sera reprise par Davy en Grande-Bretagne et par Berzelius en Suède. Ils lui donneront la caution de leur grande autorité scientifique.

A partir de 1837, C. Sprengel puis J. von Liebig (1840), J.B. Dumas et J.B. Boussingault (1841), H. de Gasparin (1843) puis Lawes et Gilbert, puis Wigman et Polstorf, etc... etc... démontreront les uns après les autres la réalité de la "Théorie minérale " de l'alimentation végétale.

Il faudra attendre la fin du siècle pour que Schloesing montre le rôle physique de l'humus et que les microbiologistes Beijerinck, Omeliansky mettent en évidence les mécanismes de son action comme stock d'éléments fertilisants.

Sur un plan sémantique on se doit de constater que, jusqu'à nos jours, le mot humus conservera sa double signification et de constituant et d'horizon (comme l'atteste l'usage courant des expressions telles que "humus doux", "humus brut ", humus de type "mull ", "moder", etc...). On pourrait ajouter, et de manière fort schématique, que la signification qui lui est attribuée dépend du "contexte scientifique :

- «humus-constituant " pour les chimistes et les agronomes;

— «humus-horizon" pour les pédologues (pédogénéticiens) et les forestiers.

$C^{\prime}$ 'est ainsi qu'à la fin du XIX siècle, les ouvrages de chimie agricole (par exemple Grandeau, 1879) traitent de l'“ humus-constituant " en même temps que le forestier danois Müller édite, en français, en 1889, son extraordinaire livre (précurseur des études pédogénétiques modernes), "Recherches sur les formes naturelles de l'humus et leur influence sur la végétation et le sol" qui concerne l'«humus-horizon". Cette ambiguité de vocabulaire se perpétue de nos jours comme le souligne très nettement Duchaufour (1970) à l'article humus de l'Encyclopédie Universalis (volume 8, p. 610):

"... le mot "humus" désigne dans son sens le plus étroit, la fraction de la matière organique du sol qui a subi une transformation plus ou moins rapide, d'origine biologique et aussi physicochimique... Au sens le plus large du terme "... (l'humus)..." désigne l'ensemble des horizons organiques... "(14).

Mais dès 1849 , le mot humus entrait en littérature: "là commence l'humus de l'Ukrayne, une terre grasse et noire d'une profondeur de cinquante pieds, qu'on ne fume jamais et que l'on sème toujours de blé..." (H. de Balzac, lettres de Kiev, 1849, œuvres complètes, tome III, p. 681).

(13) Substance noirâtre extraite par Vauquelin (1797) de l'écorce d'Orme (Uimus en latin) à l'aide d'alcalis, le terme "ulmine" ayant été proposé soit par Klaproth en 1804 (selon Maillard, 1913), soit par Thomson en 1807 (selon Kononova, 1961).

(14) Soulignès par nous-mêmes (C.F. et J.B.). 


\section{CONCLUSIONS}

Le mot humus réapparaît dans le langage scientifique vers la moitié du XVIII' siècle avec le sens de "terre végétale" (Diderot, 1755). II lui faudra au moins trente ans (Abbé Rozier, 1781) pour commencer à ètre diffusè avec d'ailleurs des significations variables (et parfois non prècisèes) telles que sol, horizon organique ou organo-minéral, constituant organique du sol. Ce n'est qu'à partir de 1800 que l'on peut considérer son emploi dans les écrits scientifiques comme courant, et c'est probablement Thaer (1809) qui lui attribue très précisément la signification de constituant. Il conservera, toutefois, jusqu'à nos jours, sa double signification d'horizon et de constituant. C'est, bien évidemment, à cette même époque (début XIX $X^{e}$ siècle) que naît la "chimie de l'humus", sans que pour autant apparaissent rapidement les qualificatifs "humine " et " humique ". Mais ceci est une autre histoire !
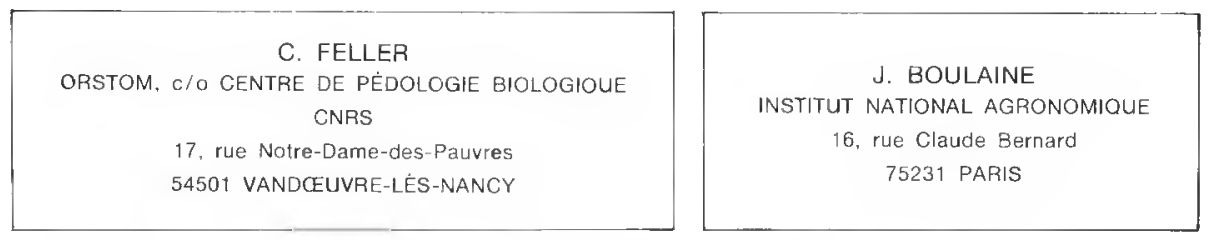

Remerciements: Nous remercions vivement M. Ph. DUCHAUFOUR pour ses avis et informations.

\section{BIBLIOGRAPHIE}

Anonyme. - Corps d'observation de la Socièté d'agriculture, de commerce et des arts ètablie par les Ėtats de Bretagne en 1759 et 1760 . - Paris : B. Brunet, 1762. - $359 \mathrm{p}$.

Anonyme. - Dictionnaire domestique portatif. - 3 tomes, - Paris : Vincent, 1765.

Anonyme. - Histoire de l'agriculture ancienne extraite de l'histoire naturelle de Pline, livre 18. - Paris: G. Despres, 1765 . - $358 \mathrm{p}$.

Anonyme. - Mèmoire sur les défrichements. - $2^{\circ}$ eddition. - Paris : Veuve d'Houry, 1761. - 348 p.

Anonyme. - Pratique des défrichements. - Paris: Veuve d'Houry, $1760 .-151 \mathrm{p}$.

Anonyme. - Le spectacle de la nature ou entretiens sur les particularites de l'histoire naturelle. $2^{e}$ partie tome 2, nouvelle édition - Beauvais: Veuve Estienne et Jean Desaint, 1735 . - $488 \mathrm{p}$.

BERGMANN. - ... Dissertation. Quels sont les caracteres principaux des terres en gèneral ? In : Assemblẻe publique de la Société royale des Sciences, tenue à Montpellier le 8 décembre 1773 . - Montpellier: J. Martel, 1773. - 41 p.

BONNET (C.). - Contemplation de la nature. - 2 tomes. - Amsterdam : M.M. Roy, 1764.

BOURDE (A.). - Agronomies et agronomes en France au XVII siècle. - Tomes I, II et III, 1962.

BUFFON (Cte de). - Histoire naturelle gènèrale et particulière avec la description du cabinet du Roy. $4^{*}$ édition. - Paris : Royale, 1752. - Tome 1, $451 \mathrm{p}$.

CHAPTAL (Cte de). - Chimie appliquèe à l'agriculture (tome 1, p. 43). - Paris : Huzard, 1823.

DELAILLEVAULT. - Recherches sur la houille d'engrais et les houillères. - 2 tomes. - La Haye, Paris : Servière, 1783.

DICTIONNAIRE de l'Académie française. - Avignon: J. Garrigan, 1777. Nouvelle édition.

DIDEROT, D'ALEMBERT. - Encyclopèdie ou dictionnaire raisonnè des Sciences, des Arts et des Mètiers. tome 8. - Paris, 1765. 
DOUETTE-RICHARDOT (N.). - De la pratique de l'agriculture. - Paris: A.J. Marchant, 1806. - 694 p. DUCHAUFOUR (Ph.). - Article « humus», in: Encyclopédie Universalis. - Paris, 1970, volume 8, p. 610. DUHAMEL DU MONCEAU. - Élèments d'agriculture. - 2 tomes, - Paris : Guérin et Delatour, 1763.

FABRONI (Baron J.-V.). - Réflexions sur l'état actuel de l'agriculture ou exposition d'un véritable plan pour cultiver ses terres avec le plus grand avantage et pour se procurer des engrais. - Paris, 1780 (en français).

GIESECKE (F.). - Geschichtliche Uberblick über die Entwicklung der Bodenkunde... In : Handbuch der Bodenlehre/E. Blanck. - Berlin : Springer, 1929.

GODARD (D.). - Les agronomes du XVIII ${ }^{\circ}$ siècle. - Mémoire de maîtrise. - Université de Paris I, UER 10 Philosophie. - Rapport multigraphié, $123 \mathrm{p}$.

GRACE (de). - Le bon jardinier. Almanach pour l'année 1783. - Nouvelle édition. - Paris : E. Onfroy, 1783. $-384 \mathrm{p}$.

GRANDEAU (L.). - Chimie et physiologie appliquée à l'agriculture et à la sylviculture. 1. La nutrition de la plante. - Paris : Berger-Levrault et Cie, 1879. - $624 \mathrm{p}$.

HAGBERG (K.). - Carl Linné " le roi des fleurs". Traduit du suédois par T. Hammar et M. Metzger. - Paris : Ed. "Je sers", 1944. - $210 \mathrm{p}$.

HASSENFRATZ (J.U.). - Sur la nutrition des végètaux. - Ann. Chimie, 1792, tome 13, pp. 178-192 et $318-$ 330 , tome 14 , pp. 55-64.

HIRZEL. - Le socrate rustique. - Zürich: M. Barbau, 1763. - 172 p.

HOME $\left(F_{1}\right)$. - Les principes de l'agriculture et de la végétation. - Traduit de l'anglais. — Paris : Prault, 1761. $-301 \mathrm{p}$.

INGEN-HOUSZ (J.). - Expériences sur les végétaux. - Paris, 1780.

KONONOVA (M.M.). - Soil organic matter, its nature, its role in soil formation and in soil fertility. $-2^{2}$ édition anglaise. - Oxford: Pergamon, 1961. - $505 \mathrm{p}$.

LA SALLE DE L'ETANG (de). - Manuel d'agriculture pour le laboureur, pour le propriétaire et pour le gouvernement. - Nouvelle édition. - Paris: P.F. Didot, 1768. - $583 \mathrm{p}$.

LIGER (L.). - La nouvelle maison rustique ou économie générale de tous les biens de campagne. - 2 tomes. $-3^{\circ}$ édition: Paris: Prudhomme, 1721, tome 1, 782 p. ; $6^{e}$ édition: Paris: Saugrain, 1749, tome 1, $1004 \mathrm{p}$.

MAILLARD (L.C.). - Genèse des matières protéiques et des matières humiques. - Paris: Masson, 1913. $423 \mathrm{p}$.

MARTIN (F.). - Les mots latins. - Paris: Hachette, 1941.

MÜLLER (P.E.). - Recherches sur les formes naturelles de l'humus et leur influence sur la végétation et le sol. - Paris, Nancy: Berger-Levrault et Cie, 1889. - $351 \mathrm{p}$.

PATRIN. - Article " humus, terre végétale ou terreau ". In : Nouveau dictionnaire d'histoire naturelle appliquée aux arts, principalement à l'agriculture et à l'économie rurale et domestique », tome 11, pp. 445-447. Paris: Crapelet, 1803.

PLAISANCE (G.), CAILLEUX (A.). - Dictionnaire des sols. - Paris : La Maison Rustique, 1958. - 604 p.

PRIESTLEY (J.). - Expérience et observation sur différentes espèces d'air. - 5 tomes. - Traduit de l'anglais par Gibelin. - Paris : Nyon, 1777.

ROBERT (P.). - Dictionnaire alphabétique et analogique de la langue française (dit le »Petit Robert "). Paris: SNL, Alain Rey, 1967. - 1970 p.

ROZIER (Abbé). - Cours complet d'agriculture thèorique, pratique, économique et de médecine rurale et vétérinaire. - 12 tomes. - Paris, 1781 à 1805

SAUSSURE (Th. de). - Recherches chimiques sur la végétation. - Paris: Nyon, 1804. - 327 p. (far, similé : Paris : Gauthiers-Villars, 1957).

SENEBIER. - Mémoires physico-chimiques sur l'influence de la lumière solaire pour modifier les êtres des trois règnes de la nature et surtout ceux du règne végétal. - 3 volumes. - 1792 .

THAER (A.). - Grunsätze der rationnellen Landwirschaft. - 4 tomes. - Berlin : Realschulbuch, 1809-1812.

THAER (A.) - - Principes raisonnès d'agriculture. - 4 tomes. - Traduit de l'allemand par E.V.B. Crud. Paris: J.J. Prechoud, 1811-1816.

TRÉSOR DE LA LANGUE FRANÇAISE (TLF). - Dictionnaire de la langue du $X^{\circ} X^{\circ}$ et du $X X^{\bullet}$ siècle. - Paris : Éditions du CNRS, 1981. - tome 9, pp. 990-991.

TULL (J.). - The horse-hoeing husbandry or an essay on the principales of tillage and vegetation. - London, 1733.

VALLEMONT (Abbé de). - Curiosités de la nature et de l'art sur la végétation ou l'agriculture et le jardinage dans leur perfection. - 2 tomes, nouvelle édition. - Paris : Societe's, 1711.

VALMONT DE BOMARE. - Dictionnaire raisonné universel d'Histoire naturelle. - Paris, 1768.

VIREY. - Article "Alimens" in: Nouveau dictionnaire d'histoire naturelle appliquée aux arts, principalement à l'agriculture et à l'économie rurale et domestique, tome 1, 1803, pp. 555-572.

WALLERIUS (J.G.). - Minéralogie ou description générale des substances du régne minéral. - tome 1 . Paris: Durand et Pissot, 1753. - $111 \mathrm{p}$.

YVART (V.). - Coup d'ceil sur le sol, le climat, l'agriculture de la France comparés avec les contrées qui l'avoisinent et particulièrement avec I'Angleterre. - Paris, 1807. - $111 \mathrm{p}$. 